\title{
This Past Year in Research
}

\author{
John Somberga, b
}

Twenty twenty-one has already been an eventful year. The events of the year have made successful cardiovascular research more difficult, but the community of researchers has persevered. Despite the pandemic we have seen many advances in basic and clinical research.

We have seen advances in the treatment of serious but less prevalent forms of heart failure and myocyte disease. For the first time we have an effective treatment for hypertrophic cardiomyopathy with mavacamten. Mavacamten is the first in its class myosin inhibitor which can aid in the reduction of the left ventricular outflow obstruction in patients with obstructive hypertrophic cardiomyopathy. Additionally tafamidis, inotersen and patisiran have been introduced for the treatment of transthyretin amyloidosis, another less common cause of heart failure but one that previously was without a targeted treatment. Of great significance is the introduction of a number of anti-diabetic (type II) agents that have significant benefit in the treatment of heart failure with reduced ejection fraction. Empagliflozin, dapagliflozin, and canagliflozin have been found to add additional benefit in reducing mortality when given on top of combined standard heart failure therapy including sacubitril/valsartan. These unexpected findings will initiate a search for the mechanism(s) explaining the effectiveness of these glucose-lowering agents in heart failure. Some have diuretic properties but diuretics have never been shown to affect mortality and thus there probably is a significant unknown mechanism that cause benefit in heart failure.

The treatment of heart failure with preserved ejection fraction has been a more difficult therapeutic problem. Recent studies with sacubitril/valsartan suggest that for patients with mildly compromised left ventricle (LV) function improvement is seen. Certainly more studies on the mechanics of failure in these patients and potential therapies are needed to extend life.

There are other surprising advances in cardiovascular research. That transcatheter valves can be as durable as surgically implanted valves is a major advance in our knowledge of valvular therapeutics. Progresses with the mitral clip and left atrial appendage closure have greatly improved treatments

Manuscript submitted December 7, 2021, accepted December 9, 2021

Published online December 11, 2021

aCardiology \& Pharmacology, Rush University, Chicago, IL 60612, USA. Email: John_Somberg@rush.edu

${ }^{b}$ Editor-in-Chief, Cardiology Research

doi: https://doi.org/10.14740/cr1344 of valvular disease and the preventing of embolic sequelae. We have gained therapeutic knowledge as how to reduce the concentration and duration of therapy with anti-platelet agents while sustaining patient benefits. Clinical studies have revealed that interventional procedures need to go beyond the stenotic lesion and look to underlying characteristics of the atherosclerotic plaques and their composition to reduce death. Spectrometric techniques, coronary ultrasound and optical coherence tomography are useful techniques. We will need to employ these techniques to a greater extent to direct stent therapy not just to reduce symptoms, but reduce myocardial infarction and death. We have become more aware that not all ischemia is due primarily to atherosclerotic obstructive disease, but may be due to vasospasm and small vessel disease. We have seen a greater recognition of the underlying pathophysiology of these conditions and thus targets for potential treatments.

Perhaps the greatest challenge to researchers this past year and to society has been the coronavirus disease 2019 (COVID-19) pandemic. The development and spread of new variants have been predictable and so far of marginal significance. However along with vaccines that need to be modified to meet new variant challenges, researchers need to develop new therapeutic agents. Agents targeted to virus replication such as protease inhibitors are on the therapeutic launching pad. We still need an organized testing program for therapeutics to be expeditiously elevated in a non-biased, thorough fashion. Clearly outpatient treatments are needed. We still do not know if ivermectin or hydroxychloroquine are effective in the early outpatient setting. Too much politics and a rush to judgement have permeated scientific discourse. Too many studies are focused in hospital, to late stages of COVID-19. We need to establish a platform for research in outpatients to quickly obtain answers on the effectiveness and safety of outpatient therapies. Twenty twenty-two is the year to accomplish this. Science with all its imperfections will succeed in supporting society and leading us to effective mitigation strategies.

\section{Acknowledgments}

None to declare.

\section{Financial Disclosure}

None to declare. 


\section{Conflict of Interest}

None to declare.

\section{Data Availability}

The author declares that data supporting the findings of this study are available within the article. 